\title{
Baseline circulating IL-17 predicts toxicity while TGF- $\beta 1$ and IL-10 are prognostic of relapse in ipilimumab neoadjuvant therapy of melanoma
}

Ahmad A. Tarhini", Haris Zahoor, Yan Lin, Usha Malhotra, Cindy Sander, Lisa H. Butterfield and John M. Kirkwood

\begin{abstract}
Background: We evaluated candidate circulating serum cytokines, chemokines and growth factors in patients with locally/regionally advanced melanoma receiving neoadjuvant ipilimumab with toxicity and clinical outcome.

Methods: Patients were treated with ipilimumab (10 mg/kg IV every 3 weeks, 2 doses) before and after surgery. XMAP multiplex serum testing for 36 functionally selected cytokines and chemokines was performed at baseline and at six weeks (following ipilimumab). Based on our prior data, the association of IL-17 and immune related colitis was tested. Serum cytokines were divided into functional groups (Th1, Th2, Regulatory, Proinflammatory) and were assessed at baseline and week 6 using sparse-group Lasso modeling to assess the association of various cytokine groups with progression free survival (PFS). The linear combination of the cytokines/chemokines in this model was then used as a risk score and a Kaplan-Meier curve was generated to examine the association of the dichotomized score and PFS.
\end{abstract}

Results: Thirty-five patients were enrolled whose staging was: IIIB (3; N2b), IIIC (30; N2c, N3), IV (2). Median follow-up was 18 months. Among 33 evaluable patients, median PFS was 11 months (95\% Cl=6.2-19.2). IL-17 was found to correlate significantly with the incidence of grade 3 diarrhea/colitis when measured at baseline $(p=0.02)$ with a trend towards significance at 6 weeks $(p=0.06)$. In the modeling analysis, at baseline, the linear combination of 2 regulatory cytokines [TGF- $\beta 1(\rho=0.19)$ and IL-10 $(\rho=-0.34)$ ] was significantly associated with PFS (HR 2.66; $p=0.035)$. No significant correlations with clinical outcomes were found in examining the week 6 cytokines.

Conclusions: Baseline IL-17 level was significantly associated with the later development of severe diarrhea/colitis while the combination of baseline TGF- $\beta 1$ and IL-10 levels were associated with therapeutic clinical outcome after neoadjuvant ipilimumab. These findings warrant further investigation and validation.

Trial registration: ClinicalTrials.gov Identifier NCT00972933.

Keywords: Melanoma, Ipilimumab, CTLA4, Neoadjuvant, Cytokines

\section{Background}

Patients with clinically detectable lymphatic metastasis (American Joint Committee on Cancer stage IIIB-C) carry a poor prognosis with a risk of relapse and death that approaches $70 \%$ at 5 years [1-3]. Complete surgical excision and regional lymph node dissection followed by adjuvant therapy with high dose interferon alfa-2b (HDI) has been the standard of care for this group of patients [1]. The host immune response is known to be relevant

\footnotetext{
*Correspondence: tarhiniaa@upmc.edu

University of Pittsburgh Cancer Institute, UPMC Cancer Pavilion, 5150 Centre Avenue (555), Pittsburgh, PA 15232, USA
}

to disease outcome in melanoma and host cytokine and cellular immune responses may potentially be used to predict prognosis and response to therapy [4-6]. A better understanding of the regulation of the host effector and suppressor cellular and cytokine elements in response to melanoma may provide valuable mechanistic clues that may be exploited to predict therapeutic response to immunotherapy, including treatment with ipilimumab.

Ipilimumab is a human immunoglobulin-G (Ig G1k) anti- cytotoxic T-lymphocyte-associated protein (CTLA)4 antibody which has been approved by the United States 
Food and Drug Administration (FDA) for the treatment of advanced inoperable melanoma based on phase III trial results [7]. However, treatment is associated with significant immune related adverse events and durable therapeutic benefits appear to be confined to a subgroup of patients. Therefore, it is of critical importance to define biomarkers that may predict clinical response and the risk of toxicity in patients treated with ipilimumab [8]. Assessment of these biomarkers in peripheral blood is particularly desirable given the facile accessibility, and ability to perform highly standardized repetitive assessments of blood biomarkers.

We conducted a neoadjuvant evaluation of ipilimumab administered at $10 \mathrm{mg} / \mathrm{kg}$ for patients with locally/regionally advanced melanoma, where we have previously reported significant clinical activity and immunomodulatory changes in the circulation and the tumor microenvironment with ipilimumab [9]. In this report, we evaluated a group of functionally selected cytokines and chemokines divided into 6 subgroups, and their association with clinical outcome. IL-17 is one of the central inflammatory cytokines upregulated in inflammatory bowel disease [10]. Therefore, we tested the association of IL-17 with the risk of immune mediated colitis after neoadjuvant ipilimumab.

\section{Patients, materials and methods \\ Patients}

All eligible patients were 18 years or older with clinically detectable local and/or regional melanoma (of cutaneous, mucosal or unknown primary site of origin). The Institutional Review Board of the University of Pittsburgh approved the study and the written informed consent that was obtained from all patients participating in the study.

\section{Study design and treatment}

Patients were required to receive two doses of ipilimu$\mathrm{mab}$ at $10 \mathrm{mg} / \mathrm{kg}$ intravenously given 3 weeks apart (induction). Surgery was planned 6-8 weeks after the initiation of ipilimumab,neoadjuvant therapy. After recovery from surgery, 2 additional doses of ipilimumab 3 weeks apart were planned (maintenance). Blood specimens for correlative studies were planned at baseline and 6 weeks (before surgery)-and then at 3, 6, 9 and 12 months or at progression.

\section{Toxicity and response assessments}

For Adverse Event (AE) reporting, the description and grading scales of NCI Common Terminology Criteria for Adverse Events version 3.0 were used. Imaging studies were carried out at baseline, after 6-8 weeks of therapy (before surgery) and then at 3 months intervals for response assessment. Responses were not confirmed due to performance of definitive surgery to render the patient free of disease.

\section{Statistical methods}

The original study design and statistical plan have previously been published [9]. This report compares the correlative cytokine and chemokine blood levels studied among patients who experienced diarrhea/colitis and those who did not, using the Wilcoxon sum rank test. Given the exploratory nature of this study, no adjustment for multiple testing was done-and the nominal $p$-values are reported. Sparse group Lasso (SGL) modeling analysis was used to evaluate the association of different subgroups of the 36 functionally selected cytokines (assessed at baseline and at week 6) with progression free survival (PFS). Cytokine levels were scaled so that all cytokines have the same scale. This method allowed us to simultaneously fit the model and select the markers and group(s) associated with benefit as defined by PFS. More importantly, it allows the incorporation of prior functional group information into the modeling. The functional group information was defined by the biological function as shown below. A regular Cox proportional hazard (CoxPH) model was fitted to the markers selected by SGL. The linear combination of the cytokines (i.e. the product of the cytokine level and the CoxPH model coefficients) was then used as the risk score. We dichotomized the risk score at the median and generated KaplanMeier (KM) survival curves to examine the association of the risk score and PFS.

Baseline descriptive statistics were performed on all evaluable patients for demographic variables, laboratory parameters, toxicity and therapeutic efficacy in relation to disease PFS. PFS were estimated by the Kaplan-Meier method.

\section{Laboratory methods and corresponding statistical analyses \\ Blood processing}

Red top vacutainer tubes (no anticoagulant) were used for serum collection and all samples were processed within $24 \mathrm{~h}$ of collection (samples received before $5 \mathrm{pm}$ were processed upon receipt, those arriving after $5 \mathrm{pm}$ were processed the following morning). Serum samples were centrifuged at $2500 \mathrm{rpm}$ for $10 \mathrm{~min}$ at $4{ }^{\circ} \mathrm{C}$ according to laboratory standard operating procedures (SOPs) and single use aliquots of each patient's sera were then stored at $-80{ }^{\circ} \mathrm{C}$. The laboratory freezers were monitored continuously for any temperature fluctuations, and maintained the samples at $-80 \mathrm{C}$.

\section{Multiplex serum cytokine analysis}

Functionally selected 36 serum cytokines were tested. These included Th1 type cytokines (IFN- $\gamma$, IL-12 (p40/p70), IL-15, IL-17, IL-2, IL-7, IP-10), Th2 (IL-13, IL-5, IL-4), proinflammatory (IL-1 $\alpha$, IL-1 $\beta$, IL-6, TNF- $\alpha$, IL-1RA, IL-2R, IL-8, CRP, IL-17, IFN- $\alpha$ ), immunoregulatory (TGF- $\beta 1$, IL10, PGE2), growth factor (VEGF, G-CSF, EGF, HGF, FGF- 
basic, GM-CSF), and other/chemokines (CCL5/RANTES, CCL3/MIP-1 $\alpha$, CCL4/MIP-1 $\beta$, CCL2/MCP-1, CXCL9/MI G, CCL11/Eotaxin). The xMAP serum assay for these cytokines was performed according to the manufacturer's protocol (BioSource International (Camarillo, CA)) as previously described [11] and laboratory SOPs, and analyzed on the Bio-Plex suspension array system (Bio-Rad Laboratories, Hercules, CA). Experimental data was analyzed using five-parametric curve fitting and assay controls included kit standards and multiplex QC controls ( R \& D Systems). Inter assay variabilities for individual cytokines were 1.0 to $9.8 \%$ and intra-assay variabilities were 3.6 to $12.6 \%$ (information provided by Biosource International and validation performed in our laboratory).

\section{Results}

Patient characteristics, treatment details, efficacy and safety

Thirty five patients were enrolled between $2 / 2010$ and $10 / 2012$. Eight patients had newly diagnosed melanoma whereas 27 patients had recurrent disease after treatment that included surgery. Twenty nine patients had cutaneous primary tumors, five mucosal and one with unknown primary melanoma. Eighteen patients had in-transit metastatic melanoma. On retrospective review, two patients demonstrated stage IV disease at baseline determined through the progression of previously questionable/undetected findings [9]. By stage, patients were classified as IIIB (3; N2b), IIIC (30; N2c, N3) and IV (2). Patient demographics and baseline disease characteristics were previously published [9]. A median of 4 cycles of ipilimumab per patient were administered with a total of 106 cycles [9]. Efficacy data has been previously reported [9]. Briefly, the median follow-up for patients at risk of progression was 17.6 months and for patients who were still alive was 16.1 months. The median PFS was 10.8 months, $95 \%$ CI $(6.2,19.2)$. The probability of PFS at 6 and
12 months was $0.72,95 \% \mathrm{CI}(0.53,0.84)$ and 0.47 , $95 \%$ CI $(0.29,0.63)$. The probability of survival at both 6 and 12 months was $0.97,95 \% \mathrm{CI}(0.78,0.99)$. AEs related to ipilimumab have been reported previously [9]. These include the immune related AEs that were considered related to ipilimumab. Twenty patients (57\%) experienced diarrhea/colitis, including $9(26 \%)$ grade 1, $6(17 \%)$ grade 2 and $5(14 \%)$ grade 3 . There were no grade 4 or higher events [9].

\section{Association of IL-17 level and diarrhea/colitis}

To identify potential circulating biomarkers of efficacy and/ or toxicity, serum samples were tested for levels of a broad array of analytes. Blood IL-17 levels at baseline were found to correlate significantly with the incidence of grade 3 diarrhea/colitis $(p=0.02)$, while this association remained with a trend towards significance at 6 weeks $(p=0.06)$. Figure 1 IL-17 was not significantly associated with all grades of colitis. We found an association between baseline IL-17 and any grade $3 \mathrm{irAE}$ (similar to colitis, which was not surprising given that over $50 \%$ of patients with irAE events had colitis), but the association was slightly less significant $(P=0.03)$. When we examined all grade irAE (including colitis, rash, hepatitis, endocrinopathies, pancreatitis), there was no significant association.

\section{Association of cytokines at baseline with PFS}

The examination of individual cytokines with clinical outcome did not reveal any significant associations with disease outcome. Previous studies have identified groups of analytes as signatures of clinical outcome measures [12-16]. Therefore, we grouped our data into general functional groups based on their characterized roles in immune modulation as listed in Materials and Methods. In modelling analysis only one group of cytokines (the regulatory group) was selected by SGL as significantly associated with PFS. With further examination of these data grouped functionally, we identified a model

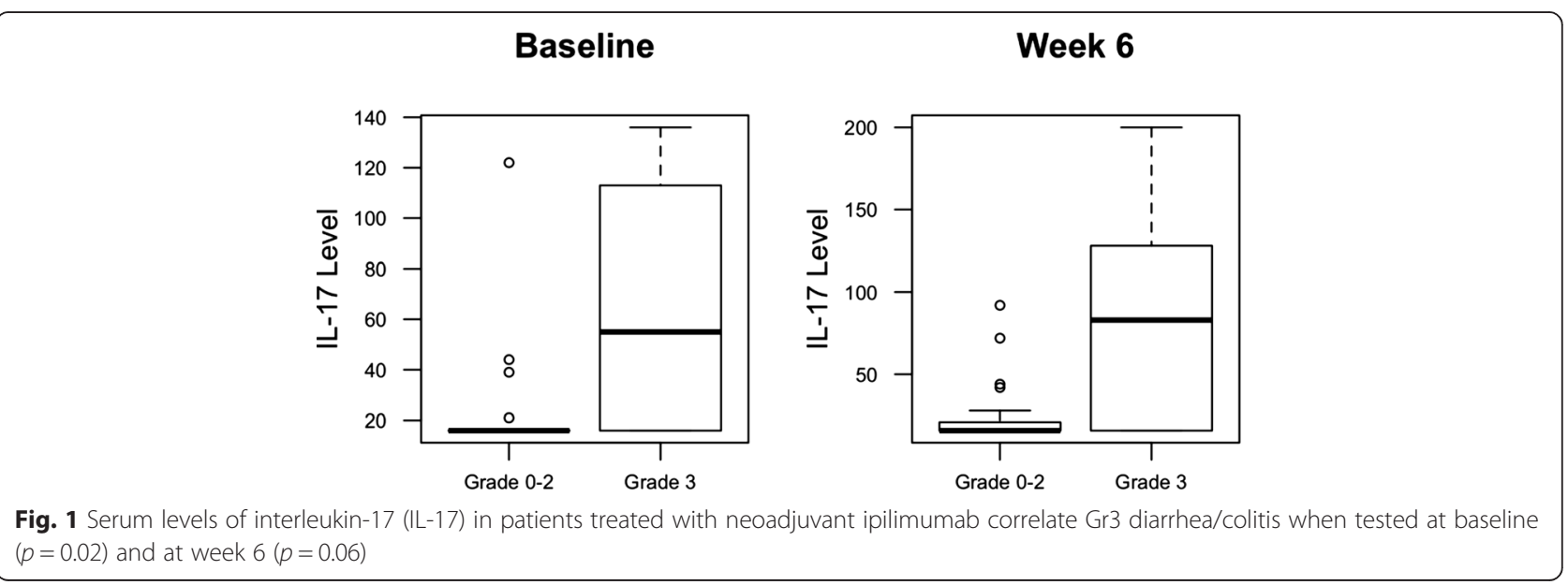


with TGF- $\beta 1$ and IL-10 as providing the best fit. IL-10 appeared to be a risk marker for progression, while unexpectedly, TGF- $\beta 1$ appeared to be a marker of nonprogression. The coefficients for the association of these two markers with PFS were 0.18 and -0.32 respectively. The dichotomized risk score based on these 2 markers was significantly associated with PFS $(p=0.036, \mathrm{HR}=2.66)$ as shown in Fig. 2. None of the type I cytokines tested correlated with clinical outcome.

\section{Association of cytokines with PFS at 6 weeks}

The examination of the correlation of blood levels of individual cytokines with clinical outcome did not reveal significant associations. Similarly, the sparsed group lasso analysis results did not reveal any combination of markers that significantly correlate with PFS.

\section{Discussion}

Ipilimumab has been shown to improve the survival of patients with advanced melanoma, but benefits only a subset of patients. Predicting the likelihood of clinical benefit from ipilimumab or the risk of developing toxicity remains an elusive goal. There is a critical need to define a biomarker or a set of biomarkers that can predict clinical response and the risk of toxicity in patients receiving ipillimumab. Major efforts in biomarker studies are ongoing and preliminary data are very encouraging, including gene expression signatures $[9,17]$, exome sequencing studies [18], and CD8 expression within the tumor microenvironment [19]. In this study, we evaluated the baseline and on-treatment serum levels of cytokines that have been functionally selected. The association of these blood cytokines with toxicity and clinical benefit after ipilimumab is novel and of potential clinical

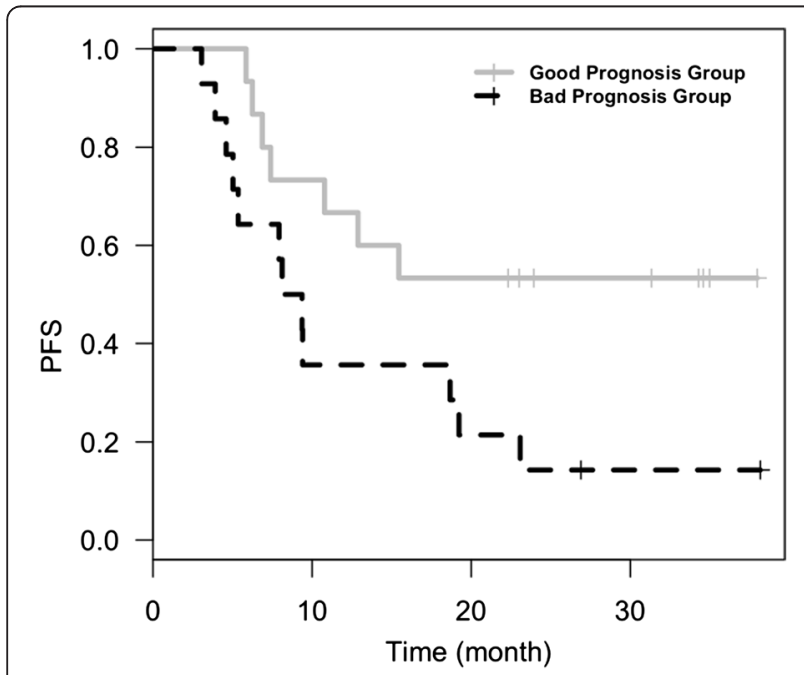

Fig. 2 Baseline serum levels of TGF- $\beta 1$ and IL-10 correlate with progression free survival (PFS) importance for this first systemic therapy to be approved in metastatic melanoma on the basis of survival benefits. Such an analysis is an important component of evaluating the host immune response to melanoma and may provide valuable mechanistic data, as well as allowing the improved prediction of benefit for patients who are considered for treatment with ipilimumab.

IL-17 is one of the central inflammatory cytokines and has been shown to be upregulated in the blood of patients with inflammatory bowel disease [10]. Therefore, the evaluation of IL-17 in patients with ipilimumabassociated inflammatory colitis is well justified. Pairwise comparisons of serum IL-17 levels in patients with colitis $(n=13)$ compared to those without immune related AEs (irAEs) $(n=16)$ demonstrated significantly higher serum IL-17 levels in patients with colitis at week 7 $(p=0.007)$ and week $12(p=0.02)$ [20]. In the present study, we observed a significant correlation between the risk of subsequent development of grade 3 immunemediated diarrhea, for the first time with circulating blood IL-17 assessed at baseline, among patients entering neoadjuvant therapy for regionally advanced melanoma. We also confirmed the prior reported association of elevated levels of IL-17 on treatment at week 6, with the development of grade 3 immune-mediated diarrhea (grade 3 was the worst grade obesreved in our study). The occurrence of irAEs has been reported to be associated with the overall clinical benefit in patients receiving ipilimumab [21-26]. This supports the notion that these AEs are related to the mechanism of action of ipilimumab, and its reversal of immune tolerance. The ability to predict a patient's risk for developing irAEs such as autoimmune colitis may significantly impact clinical care. These data support further evaluation of the blood levels of this cytokine as a predictor of risk for this $\mathrm{AE}$. Such predictive testing for inflammatory colitis may be most productive in combination with other serologic and genetic markers of inflammation known to be associated with inflammatory bowel disease in the population. To our knowledge, no associations between other cytokines and toxicity have been reported in the past.

Melanoma patients have been shown to have a different pattern of serum cytokines when compared to healthy controls $[12,27]$. Yurkovetsky et al, reported a higher level of IL-1 $\alpha$, IL-1 $\beta$, IL-6, IL-8, IL-12p40,IL-13, GM-CSF, MCP-1, MIP- $1 \alpha$, MIP-1 $\beta$, IFN- $\alpha$, TNF- $\alpha$, EGF, VEGF, and TNF-RII in the plasma of melanoma patients compared with healthy controls [12]. High serum levels of IL-1 $\beta$, IL-1 $\alpha$, IL-6, TNF- $\alpha$, and chemokines MIP- $1 \alpha$ and MIP- $1 \beta$ measured before treatment (HDI) were positively associated with RFS [12]. High levels of VEGF and fibronectin have been associated with lack of clinical response to high dose IL-2 therapy and worse overall prognosis in metastatic melanoma and renal cell carcinoma patients [28]. 
TGF $\beta$ is a well-known cytokine associated with immunosuppression [29] Blockade of TGF $\beta$ has shown therapeutic potential in preclinical tumor models and in clinical trials [29]. Our observation that higher serum TGF $\beta$ is associated with lack of tumor progression is quite unexpected. In contrast, we find that high baseline IL-10 levels to be correlated with tumor relapse. While TGF $\beta$ can have immune suppressive effects in vitro and in certain model systems in vivo [29], this observation may represent the presence of an ongoing protective immune response which is being endogenously counter-regulated in a TGF $\beta$ dependent fashion. This may be similar to our observations that increased Treg in the circulation is a positive prognostic marker (while Treg in tumors is a negative prognostic biomarker) [9]. However, our observation is not unique. A recent study reported that chemotherapy-responsive metastatic melanoma patients had higher serum TGF $\beta 1$ levels compared with chemotherapy-unresponsive patients $(p=0.05)$ [30]. In addition, patients with elevated serum TGF- $\beta 1$ concentrations had a trend towards a favorable overall survival outcome compared to those with lower levels (median 30.1 vs. 20.9 months, respectively) in that study [30]. Similarly, high serum TGF- $\beta 1$ level was found to be associated with improved survival in patients with breast cancer [31]. Therefore, this finding warrants further investigation.

We grouped serum cytokines functionally and conducted a modeling analysis in order to evaluate the linear combination of combinations of multiple cytokines belonging into four different functional groups, and to evaluate the impact of ipilimumab more rationally. The findings of our study should be taken as hypothesisgenerating since further studies are needed to validate our findings and to refine our understanding of the role that readily assessed circulating peripheral blood cytokines may play in predicting the clinical impact of immunotherapy in patients with melanoma, both toxic and therapeutic. As with any assessment of serum or plasma-based biomarkers, pre-analytic variables of time between blood draw and processing, tube type, as well as storage conditions and freeze/thaw cycles can impact results. Our single institution study utilized a CAP/ CLIA central laboratory which participates in external proficiency testing and which has substantial expertise in this area to reduce as many variables as possible. The ongoing intergroup phase III trial E1609 led by ECOG-ACRIN in which ipilimumab is being evaluated as postoperative adjuvant therapy for melanoma at $3 \mathrm{mg} / \mathrm{kg}$ and $10 \mathrm{mg} / \mathrm{kg}$ in comparison to high dose interferon- $\alpha$ provides an opportunity to further investigate and validate these results.

\section{Conclusions}

In patients with regionally advanced melanoma who enrolled in a trial of neoadjuvant therapy with ipilimumab at
$10 \mathrm{mg} / \mathrm{kg}$, baseline pretreatment IL-17 is here for the first time shown to be significantly associated with the risk of subsequent development of severe immune-mediated diarrhea. The baseline levels of TGF $\beta 1$ and IL-10, as a dichotomized risk score based on these 2 markers, are significantly associated with PFS. The observed prognostic role of high TGF $\beta 1$ levels (unlike those of IL10) is supported by other reports in the literature. These findings warrant further investigation and confirmation in larger trials.

\section{Abbreviations}

CRP: C-reactive Protein; CCL: Chemokine (C-C motif) ligand; ECOG: Eastern Cooperative Oncology Group; ACRIN: the American College of Radiology Imaging Network; EGF: Epidermal Growth Factor; FDA: Food and Drug Administration; HDI: High dose interferon alfa-2b; IL: Interleukin;

IFN: Inferferon; GM-CSF: Granulocyte Macrophage Colony-Stimulating Factor; MCP-1: Monocyte Chemoattractant Protein-1; MIP: Macrophage Inflammatory Protein; PFS: Progression Free Survival; TGF: Transforming Growth Factor; TNF: Tumor necrosis factor; VEGF: Vascular Endothelial Growth Factor.

\section{Competing interests}

Haris Zahoor, Yan Lin, Usha Malhotra, Cindy Sander and Lisa Butterfield declare that they have no competing interests.

Ahmad Tarhini and John Kirkwood have acted as consultants and have received research grant support from Bristol Myers Squibb.

\section{Authors' contributions}

Study Design: AT, UM. Study Conduct: AT, HZ, LHB, YL, CS, JMK. Data Analysis: AT, HZ, LHB, YL. Manuscript Write Up: AT, HZ, LHB, YL, CS, UM, JMK. Data Collection: AT, HZ, LHB. Final Approval of Manuscript: AT, HZ, LHB, YL, $\mathrm{CS}, \mathrm{UM}, \mathrm{JMK}$

\section{Acknowledgements}

This study was supported by NIH award P50CA121973 and by a grant from Bristol-Myers Squibb. It was also partly supported by a donation from the family our patient "Alfred Gabriel". UPCI shared resources (including the Immunologic Monitoring and Cellular Products Laboratory) that are supported in part by $\mathrm{NIH/NCl}$ award P30CA047904 were used for this project. Its content is solely the responsibility of the authors and does not necessarily represent the official views of the National Cancer Institute.

Received: 19 May 2015 Accepted: 10 July 2015

Published online: 15 September 2015

\section{References}

1. Balch CM, Urist MM, Karakousis CP, Smith TJ, Temple WJ, Drzewiecki K, et al. Efficacy of 2-cm surgical margins for intermediate-thickness melanomas (1 to $4 \mathrm{~mm}$ ). Results of a multi-institutional randomized surgical trial. Ann Surg. 1993;218:262-7. discussion 7-9.

2. Balch CM, Soong SJ, Smith T, Ross MI, Urist MM, Karakousis CP, et al. Long-term results of a prospective surgical trial comparing $2 \mathrm{~cm}$ vs. $4 \mathrm{~cm}$ excision margins for 740 patients with 1-4 mm melanomas. Ann Surg Oncol. 2001;8:101-8

3. Karakousis CP, Balch CM, Urist MM, Ross MM, Smith TJ, Bartolucci AA. Local recurrence in malignant melanoma: long-term results of the multiinstitutional randomized surgical trial. Ann Surg Oncol. 1996;3:446-52.

4. Tarhini AA, Butterfield LH, Shuai $Y$, Gooding WE, Kalinski P, Kirkwood JM. Differing patterns of circulating regulatory $T$ cells and myeloid-derived suppressor cells in metastatic melanoma patients receiving anti-CTLA4 antibody and interferon-alpha or TLR-9 agonist and GM-CSF with peptide vaccination. J Immunother. 2012;35:702-10.

5. Tarhini AA, Lin Y, Yeku O, LaFramboise WA, Ashraf M, Sander C, et al. A four-marker signature of TNF-RII, TGF-alpha, TIMP-1 and CRP is prognostic of worse survival in high-risk surgically resected melanoma. J Transl Med. 2014;12:19.

6. Tarhini AA, Shin D, Lee SJ, Stuckert J, Sander CA, Kirkwood JM. Serologic evidence of autoimmunity in E2696 and E1694 patients with high-risk melanoma treated with adjuvant interferon alfa. Melanoma Res. 2014;24:150-7. 
7. Hodi FS, O'Day SJ, McDermott DF, Weber RW, Sosman JA, Haanen JB, et al. Improved survival with ipilimumab in patients with metastatic melanoma. N Engl J Med. 2010;363:711-23.

8. Pennock GK, Waterfield W, Wolchok JD. Patient Responses to Ipilimumab, a novel immunopotentiator for metastatic melanoma: how different are these from conventional treatment responses? Am J Clin Oncol. 2012;35:606-11.

9. Tarhini AA, Edington $H$, Butterfield $L H$, Lin $Y$, Shuai $Y$, Tawbi $H$, et al. Immune monitoring of the circulation and the tumor microenvironment in patients with regionally advanced melanoma receiving neoadjuvant ipilimumab. PLoS One. 2014;9, e87705

10. Abraham C, Cho J. Interleukin-23/Th17 pathways and inflammatory bowel disease. Inflamm Bowel Dis. 2009;15:1090-100.

11. Butterfield LH, Potter DM, Kirkwood JM. Multiplex serum biomarker assessments: technical and biostatistical issues. J Transl Med. 2011;9:173.

12. Yurkovetsky ZR, Kirkwood JM, Edington HD, Marrangoni AM, Velikokhatnaya L, Winans MT, et al. Multiplex analysis of serum cytokines in melanoma patients treated with interferon-alpha2b. Clin Cancer Res. 2007;13:2422-8.

13. Wittke F, Hoffmann R, Buer J, Dallmann I, Oevermann K, Sel S, et al. Interleukin 10 (IL-10): an immunosuppressive factor and independent predictor in patients with metastatic renal cell carcinoma. Br J Cancer. 1999;79:1182-4.

14. Tartour E, Dorval T, Mosseri V, Deneux L, Mathiot C, Brailly H, et al. Serum interleukin 6 and C-reactive protein levels correlate with resistance to IL-2 therapy and poor survival in melanoma patients. Br J Cancer. 1994;69:911-3.

15. Mouawad R, Rixe O, Meric JB, Khayat D, Soubrane C. Serum interleukin-6 concentrations as predictive factor of time to progression in metastatic malignant melanoma patients treated by biochemotherapy: a retrospective study. Cytokines Cellular Mol Ther. 2002;7:151-6.

16. Dummer W, Becker JC, Schwaaf A, Leverkus M, Moll T, Brocker EB. Elevated serum levels of interleukin-10 in patients with metastatic malignant melanoma. Melanoma Res. 1995;5:67-8.

17. Tarhini AA, Lin Y, Lin HM, Sander C, Framboise WA, Kirkwood JM. Immune related melanoma gene expression profile predicts neoadjuvant ipilimumab clinical benefit. AACR Annual Meeting. 2014.

18. Snyder A, Makarov V, Merghoub T, Yuan J, Zaretsky JM, Desrichard A, et al. Genetic basis for clinical response to CTLA-4 blockade in melanoma. N Engl J Med. 2014;371:2189-99.

19. Tumeh PC, Harview CL, Yearley JH, Shintaku IP, Taylor EJ, Robert L, et al. PD-1 blockade induces responses by inhibiting adaptive immune resistance. Nature. 2014;515:568-71.

20. Callahan MK, Yang A, Tandon S, Xu Y, Wolchok JD. Evaluation of serum IL-17 levels during ipilimumab therapy: Correlation with colitis. J Clin Oncol. 2011;29(suppl; abstr 2505):2011.

21. Weber JS, O'Day S, Urba W, Powderly J, Nichol G, Yellin M, et al. Phase I/II study of ipilimumab for patients with metastatic melanoma. J Clin Oncol. 2008;26:5950-6.

22. Attia P, Phan GQ, Maker AV, Robinson MR, Quezado MM, Yang JC, et al Autoimmunity correlates with tumor regression in patients with metastatic melanoma treated with anti-cytotoxic T-lymphocyte antigen-4. J Clin Oncol. 2005;23:6043-53.

23. Phan GQ, Yang JC, Sherry RM, Hwu P, Topalian SL, Schwartzentruber DJ, et al. Cancer regression and autoimmunity induced by cytotoxic $T$ lymphocyte-associated antigen 4 blockade in patients with metastatic melanoma. Proc Natl Acad Sci U S A. 2003;100:8372-7.

24. Blansfield JA, Beck KE, Tran K, Yang JC, Hughes MS, Kammula US, et al. Cytotoxic T-lymphocyte-associated antigen-4 blockage can induce autoimmune hypophysitis in patients with metastatic melanoma and renal cancer. J Immunother. 2005;28:593-8.

25. Beck KE, Blansfield JA, Tran KQ, Feldman AL, Hughes MS, Royal RE, et al. Enterocolitis in patients with cancer after antibody blockade of cytotoxic T-lymphocyte-associated antigen 4. J Clin Oncol. 2006;24:2283-9.

26. Downey SG, Klapper JA, Smith FO, Yang JC, Sherry RM, Royal RE, et al. Prognostic factors related to clinical response in patients with metastatic melanoma treated by CTL-associated antigen-4 blockade. Clin Cancer Res. 2007;13:6681-8

27. Shetty G, Beasley GM, Sparks S, Barfield M, Masoud M, Mosca PJ, et al. Plasma cytokine analysis in patients with advanced extremity melanoma undergoing isolated limb infusion. Ann Surg Oncol. 2013;20:1128-35.
28. Sabatino M, Kim-Schulze S, Panelli MC, Stroncek D, Wang E, Taback B, et al. Serum vascular endothelial growth factor and fibronectin predict clinical response to high-dose interleukin-2 therapy. J Clin Oncol. 2009;27:2645-52.

29. Patil AS, Sable RB, Kothari RM. An update on transforming growth factor-beta (TGF-beta): sources, types, functions and clinical applicability for cartilage/bone healing. J Cell Physiol. 2011;226:3094-103.

30. Tas F, Karabulut S, Yasasever CT, Duranyildiz D. Serum transforming growth factor-beta 1 (TGF-beta1) levels have diagnostic, predictive, and possible prognostic roles in patients with melanoma. Tumour Biol. 2014;35:7233-7.

31. Ciftci R, Tas F, Yasasever CT, Aksit E, Karabulut S, Sen F, et al. High serum transforming growth factor beta 1 (TGFB1) level predicts better survival in breast cancer. Tumour Biol. 2014;35:6941-8.

\section{Submit your next manuscript to BioMed Central and take full advantage of:}

- Convenient online submission

- Thorough peer review

- No space constraints or color figure charges

- Immediate publication on acceptance

- Inclusion in PubMed, CAS, Scopus and Google Scholar

- Research which is freely available for redistribution 